\title{
AN ATLAS OF MIXED-MORPHOLOGY PAIRS
}

\author{
N. A. Sharp \\ National Optical Astronomy Observatories \\ P.O.Box 26732, Tucson AZ 85726-6732
}

Current theories of galaxy formation imply that environment is the most important factor in deciding whether a collapsing region becomes a spiral galaxy or an elliptical galaxy. If this is the case, then isolated pairs comprising a galaxy of each morphology provide an especially interesting test, since, strictly speaking, they should not exist.

The first phase of studying E-S pairs is to define a sample which is genuinely isolated and genuinely $\mathrm{E}-\mathrm{S}$. The catalog compiled by Karachentsev is the most useful northern hemisphere starting point, containing 603 pairs which obeyed certain well-defined angular isolation criteria. All of these pairs now have velocities, enabling further removal of at least some of the non-physical binaries. The conventional cutoff at $500 \mathrm{~km} \mathrm{~s}^{-1}$ was used for this purpose.

However, the morphological types were estimated from Palomar Sky Survey prints, and are consequently not very accurate. It was therefore decided to obtain good CCD images in the B-band of all pairs from the Karachentsev sample which were classified as either E-S (73 pairs) or E-S0 (91 pairs), for a total of 164 pairs. This includes the 24 pairs with known redshift differences greater than $500 \mathrm{~km} \mathrm{~s}^{-1}$ (9 E-S and $\left.15 \mathrm{E}-\mathrm{S} 0\right)$, which were also imaged in this survey, not only for completeness but also in case the criteria were later changed, or the redshifts were found to be in error.

A total of 198 CCD frames were obtained between February 1986 and the present, with most of the observations being completed by the summer of 1987. A number of missing 'stragglers' and replacements for poor quality images were taken more recently. Most observations used the \#1$0.9 \mathrm{~m}$ telescope at Kitt Peak, with a few frames from the $2.1 \mathrm{~m}$. Some pairs required more than one image due to their separation. The morphological study did not require photometric conditions, but calibrations were made for almost all images anyway, and the weather was probably photometric at least half of the time, at least to an accuracy of around 0.1 magnitudes.

The results of this first phase will be published in the form of an Atlas, from which any interested readers will be able to select their own sub-sample using their preferred morphological criteria. Preliminary classifications suggest that less than half of the 140 physical pairs are actually genuine E-S systems, with about 35 certain pairs, and about the same number of less certain systems (Irregular rather than S, possibly S0 rather than E). Some of these uncertainties will be settled with further analysis of the existing data (e.g., using calibrated radial profiles), but some will require further observations.

Once the sample has been refined to genuine E-S pairs, further photometric data will be obtained, in at least the B and V pass-bands. Kinematical data may also be useful. The properties of the refined sample will then be compared to those of 'regular' field galaxies.

Fig. 1: Example of planned atlas page

In each picture, the lower scale bar is one arc minute in length when solid, and 0.5 arc minutes in length when dashed. The upper scale bar is $10 \mathrm{kpc}$ in length, based on the mean redshift and a Hubble constant of $100 \mathrm{~km} \mathrm{~s}^{-1} \mathrm{Mpc}^{-1}$ (dashed bars would be $5 \mathrm{kpc}$, if the image scale was such that $10 \mathrm{kpc}$ did not fit). Pairs with a redshift difference in excess of $500 \mathrm{~km} \mathrm{~s}^{-1}$ do not have the physical scale bar included. All binaries are identified by their number in the Karachentsev catalog. All pictures have North up and East to the left. 


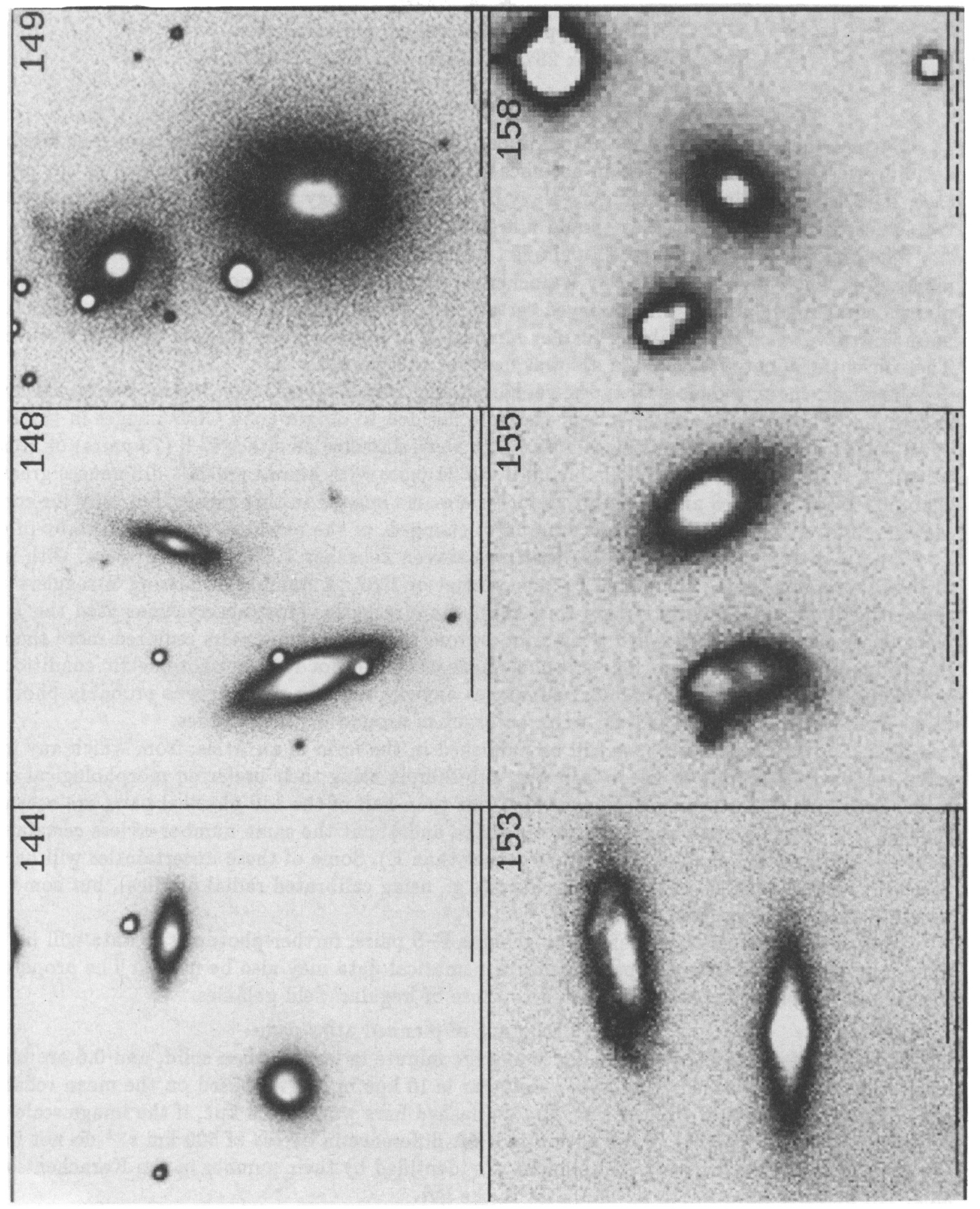

